\title{
Canonical features of human antibodies recognizing the influenza hemagglutinin trimer interface
}

\author{
Seth J. Zost, ${ }^{1,2}$ Jinhui Dong, ${ }^{1}$ Iuliia M. Gilchuk, ${ }^{1}$ Pavlo Gilchuk, ${ }^{1}$ Natalie J. Thornburg, ${ }^{1}$ Sandhya Bangaru, ${ }^{2}$ Nurgun Kose, ${ }^{1}$ \\ Jessica A. Finn, , ${ }^{1,2}$ Robin Bombardi, ${ }^{1}$ Cinque Soto, ${ }^{1,4}$ Elaine C. Chen, ${ }^{1,2}$ Rachel S. Nargi, ${ }^{1}$ Rachel E. Sutton, ${ }^{1}$ Ryan P. Irving, ${ }^{1}$ \\ Naveenchandra Suryadevara, ${ }^{1}$ Jonna B. Westover, ${ }^{3}$ Robert H. Carnahan, ${ }^{1,4}$ Hannah L. Turner, ${ }^{5}$ Sheng Li, ${ }^{6}$ \\ Andrew B. Ward, ${ }^{5}$ and James E. Crowe Jr. ${ }^{1,2,4}$ \\ TThe Vanderbilt Vaccine Center and ${ }^{2}$ Department of Pathology, Microbiology, and Immunology, Vanderbilt University Medical Center, Nashville, Tennessee, USA. ${ }^{3}$ Department of Animal, Dairy, and Veterinary \\ Sciences, Utah State University, Logan, Utah, USA. ${ }^{4}$ Department of Pediatrics, Vanderbilt University Medical Center, Nashville, Tennessee, USA. ${ }^{5}$ Department of Integrative Structural and Computational \\ Biology, The Scripps Research Institute, La Jolla, California, USA. ${ }^{6}$ Department of Medicine and Biomedical Sciences, School of Medicine, University of California, San Diego, California, USA
}

\begin{abstract}
Broadly reactive antibodies targeting the influenza A virus hemagglutinin (HA) head domain are thought to be rare and to require extensive somatic mutations or unusual structural features to achieve breadth against divergent HA subtypes. Here we describe common genetic and structural features of protective human antibodies from several individuals recognizing the trimer interface (TI) of the influenza A HA head, a recently identified site of vulnerability. We examined the sequence of TI-reactive antibodies, determined crystal structures for TI antibody-antigen complexes, and analyzed the contact residues of the antibodies on HA to discover common genetic and structural features of $\mathrm{TI}$ antibodies. Our data reveal that many TI antibodies are encoded by a light chain variable gene segment incorporating a shared somatic mutation. In addition, these antibodies have a shared acidic residue in the heavy chain despite originating from diverse heavy chain variable gene segments. These studies show that the TI region of influenza A HA is a major antigenic site with conserved structural features that are recognized by a common human B cell public clonotype. The canonical nature of this antibody-antigen interaction suggests that the TI epitope might serve as an important target for structure-based vaccine design.
\end{abstract}

\section{Introduction}

Influenza A virus (IAV) is one of the most common causes of severe lower respiratory illness in humans and exhibits a wide antigenic diversity in circulating field strains. Seasonal epidemics with $\mathrm{H} 1$ and H3 IAV subtypes occurs yearly, and other zoonotic IAVs with H1, H3, H5, H6, H7, H9, and H10 HAs cause outbreaks of human infections sporadically $(1,2)$. Incomplete matches of the seasonal IAV vaccine strains with the antigenically drifted viruses causing epidemics lead to vaccine ineffectiveness and contribute to severe influenza seasons (3-5).

A high priority for current IAV vaccine development is the development of vaccine antigens that induce broad and protective immune responses $(6,7)$. Antibodies to the hemagglutinin (HA) stem domain can exhibit heterosubtypic recognition patterns (8-21). However, the accessibility of the stem domain

Authorship note: SJZ and JD contributed equally to this work.

Conflict of interest: JEC has served as a consultant for Luna Biologics, is a member of the Scientific Advisory Board of Meissa Vaccines, and is the founder of IDBiologics. The Crowe laboratory at Vanderbilt University Medical Center has received sponsored research agreements from Takeda Vaccines, IDBiologics, and AstraZeneca. SJZ and JEC are inventors on a patent filed by Vanderbilt University ("Human monoclonal antibodies to a new universal influenza $A$ hemagglutinin head domain epitope," U.S provisional patent application serial no. 63/137,458).

Copyright: (5) 2021, American Society for Clinical Investigation.

Submitted: December 9, 2020; Accepted: June 16, 2021; Published: August 2, 2021.

Reference information: J Clin Invest. 2021;131(15):e146791.

https://doi.org/10.1172/JCl146791. may be reduced on the virion particle, and stem-specific antibodies exhibit mostly a moderate protective capacity. The HA head domain is immunodominant and is the target of most antibody responses induced by IAV vaccine or infection (22-26), but head domain-specific antibodies often recognize a narrow spectrum of IAVs. Recently, several groups have identified a class of human antibodies recognizing a highly conserved region on the HA head domain, the trimer interface (TI) region (27-29). The few monoclonal antibodies (mAbs) reported have broad recognition patterns for diverse IAVs, are nonneutralizing, and protect in animal models (27-29). The critical HA residues recognized by the TI mAbs reported to date remain conserved across most subtypes of influenza A viruses, suggesting that the TI site might serve as an attractive antigenic target for epitope-based universal IAV vaccine design. However, designing vaccine antigens based on recognition of unusual antibodies isolated only from rare individuals is not desirable, since universal influenza vaccine antigens should possess the capacity to induce broadly protective antibodies in a large number of individuals with diverse exposure histories. Here, we describe 5 new TI mAbs from 4 independent lineages, isolated from 3 separate individuals. Remarkably, we found that these and many other TI antibodies are members of a common public B cell clonotype with canonical genetic and structural features, suggesting that most human subjects have the capacity to make TI antibodies with a minimal number of somatic mutations needed to accomplish near-universal influenza A recognition. 
A

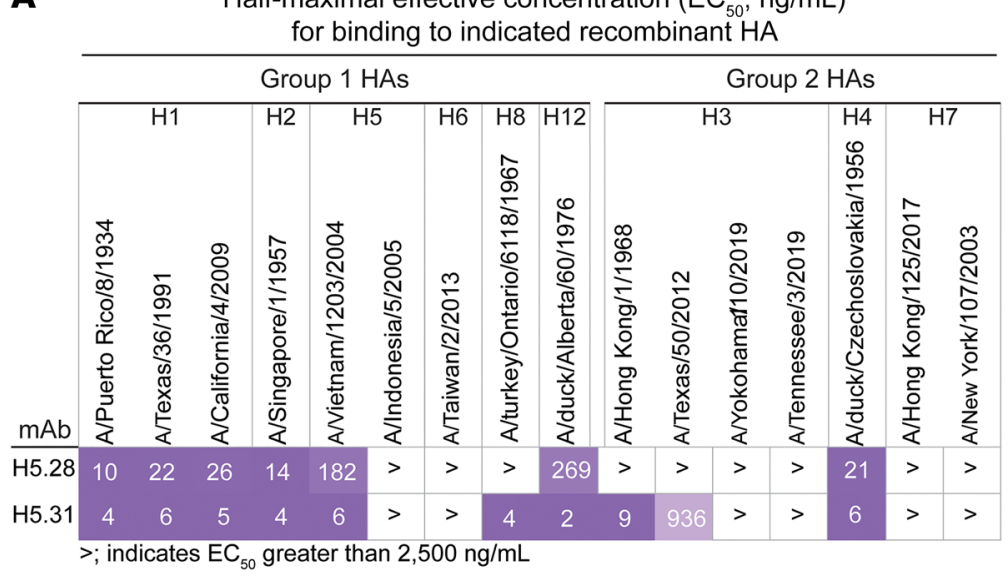
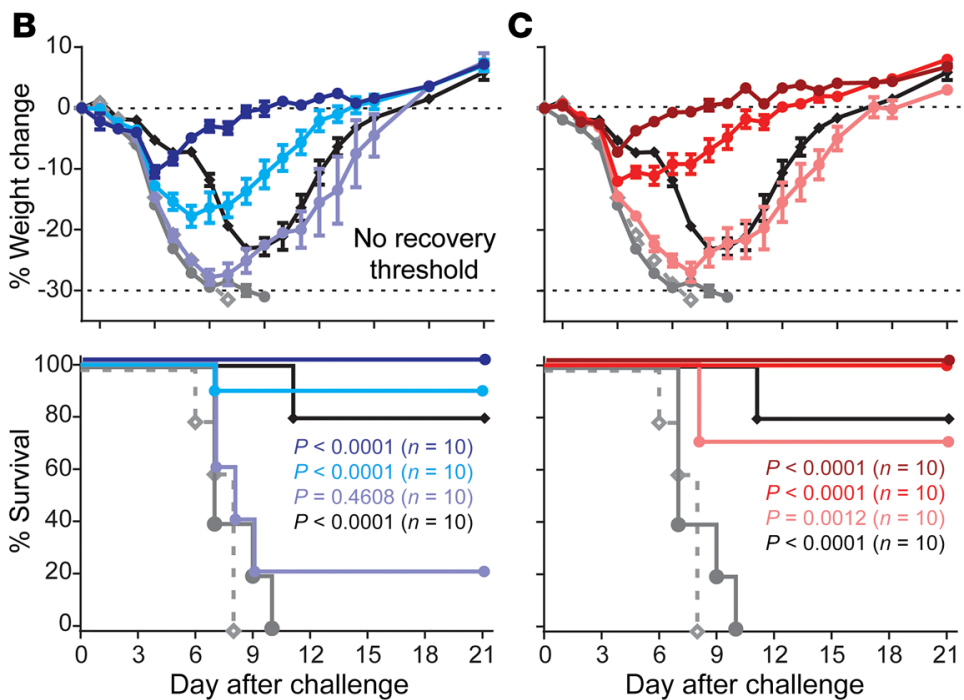

Figure 1. MAb H5.28 and H5.31 cross-react broadly and protect in vivo. (A) ELISA to determine strength of binding of $\mathrm{H} 5.28$ or $\mathrm{H} 5.31$ to a panel of recombinant $\mathrm{HAs} \mathrm{EC}_{50}$ values for binding $(\mathrm{ng} / \mathrm{mL})$ are shown in each square. The purple and white color scale indicates strength of binding, with darker squares indicating strong binding. Body-weight changes and survival in mice that received prophylactic treatment with mAb H5.28 (B, blue lines) or mAb H5.31 (C, red lines) at 3 different doses. Mice were challenged intranasally with a lethal dose (2200 CCID $_{50}$ ) of A/California/04/2009 virus 24 hours after prophylactic administration. A negative control group was treated with the anti-dengue virus MAb DENV 2D22 (solid gray lines), while a positive control group was treated with oseltamivir daily for 5 days (black lines). An additional control group received a PBS placebo (dashed gray lines). The controls shown in both $\mathbf{B}$ and $\mathbf{C}$ represent the same experimental groups. For weight loss curves, error bars show the SEM. Survival curves were estimated using the Kaplan-Meier method and each treatment group was compared with the DENV 2 222 group using a 2-sided log rank (Mantel-Cox) test. $P$ values are indicated for each comparison, and a Bonferroni-corrected threshold for statistical significance was set to $P$ less than 0.006 .

\section{Results}

Identification of broadly reactive human TI $m A$ bs in a panel of $\mathrm{H} 5$ $H A$-specific mAbs. We previously reported isolation of H5 HA-specific human antibodies from otherwise healthy subjects who had received an A/Vietnam/1203/2004 H5N1 (VN/04) subunit vaccine (30). Here we examined the reactivity of some of the H5reactive mAbs to determine if any exhibited heterosubtypic breadth of recognition for diverse HA subtypes. To investigate the breadth, we tested purified IgGs for binding activity to HA from different IAV subtypes. All HA proteins used were recombinant trimers. mAbs designated H5.28 and H5.31 exhibited breadth of binding to recombinant HAs from Group 1 and Group 2 (Figure 1A). DNA encoding the WT H5.28 and H5.31 variable regions were synthesized and recombinant forms of IgG proteins were expressed. Hybridoma-generated antibody (designated H5.28 or H5.31) was used for the assays unless the recombinant form is specified (designated as rH5.28 or rH5.31). Neither hybridoma-derived H5.28 nor hybridoma-derived H5.31 had neutralizing or hemagglutination-inhibiting activity for a VN/04 H5N1 virus when tested in concentrations as high as $10 \mu \mathrm{g} / \mathrm{mL}$. Recombinant purified IgG molecules made in $293 \mathrm{~F}$ or Chinese hamster ovary (CHO) cells also lacked neutralizing and hemagglutination-inhibiting activity (Supplemental Figure 1; supplemental material available online with this article; https://doi.org/10.1172/JCI146791DS1). Despite the fact that $\mathrm{H} 5.28$ and $\mathrm{H} 5.31$ did not neutralize virus in vitro, we tested whether these mAbs protected against weight loss and death in mice following a stringent lethal challenge with a mouse-adapted A/California/04/2009 (H1N1pdm) strain. BALB/c mice $(n=10$ per group) were administered 1,3 , or $10 \mathrm{mg} / \mathrm{kg}$ of $\mathrm{H} 5.28$ or H5.31 IgG or a similarly prepared control antibody by the intraperitoneal route, and then challenged by the intranasal route 24 hours later with a lethal dose of virus. H5.28 or H5.31 mediated dose-dependent protection against mortality and protection against severe weight loss when administered prophylactically at 3 tested doses. Protection with low-dose mAb treatment was comparable to that of high-dose oseltamivir given at a high daily dose of $30 \mathrm{mg} / \mathrm{kg} /$ day on days 0 to 4 after virus inoculation. Mice treated with H5.28 (Figure 1B) or H5.31 (Figure 1C) ( $n=10$ for each group) showed protection from weight loss after virus challenge in a dose-dependent manner whereas mice treated with PBS or the 2D22 control anti- 
body succumbed to infection. These results indicate the ability of $\mathrm{mAb} \mathrm{H} 5.28$ or $\mathrm{H} 5.31$ to protect in vivo against lethal virus challenge using a virulent influenza A strain.

To identify the specific epitope recognized by these mAbs, we performed hydrogen deuterium exchange mass spectrometry (HDX-MS) experiments. We used a monomeric head domain of $\mathrm{H} 5$ (based on strain VN/04) to identify peptides on the surface of HA that are occluded following binding of H5.28. We found that $\mathrm{H} 5.28 \mathrm{Fab}$ reduced deuterium labeling of peptides comprising residues 96 to 105,136 to 147 , and 217 to 233 (H3 structure numbering, Figure 2A and Supplemental Figure 2). From the HDX-MS studies, we anticipated that $\mathrm{H} 5.28$ or $\mathrm{H} 5.31$ binding to the HA trimer destabilizes the trimeric interface of native HA. To directly examine the effect of these Fabs on the HA trimer, we performed negative-stain electron microscopy (nsEM) of HA (uncleaved H1 A/ California/04/2009 stabilized with a GCN4 trimerization motif [H1 HAO]) in complex with either H5.28 or H5.31 Fab incubated for different lengths of time. Native $\mathrm{H} 1 \mathrm{HAO}$ trimer remained in its trimeric conformation during nsEM sample preparation (Figure 2B). In contrast, we observed that upon exposure to H5.28 or H5.31 even for 20 seconds the HAO trimers quickly degraded into Fabbound monomeric HA (Figure 2B). Despite extensive trials, the intermediate stage of this structural change could not be obtained, apparently due to the rapid transformation of the HAO from trimeric to monomeric states induced by antibody binding. While it is unclear exactly how this transformation occurs, one possibility is that the antibody catches an HA head domain during transient "breathing" of the HA head domains. These results demonstrate that H5.28 and H5.31 bind the uncleaved HAO trimer, and then dissociate the trimer in vitro (Figure 2B). Both mAbs H5.28 and H5.31 bound preferentially to uncleaved HA (with reduced binding to cleaved HA) on the surface of HA-transfected cells, while a recombinant form of a representative stem domain antibody bound well to both forms (Figure 2C and Supplemental Figure 3).

To determine the molecular details of the interaction of $\mathrm{H} 5.28$ and H5.31 with the TI site, crystal structures of the H5.31 or H5.28 Fabs and their complexes with the HA head domain from VN/04 were determined at 3.00 Å or 4.00 Å resolution, respectively (Supplemental Table 1). The complex structures revealed that H5.28 and $\mathrm{H} 5.31$ recognize an epitope in the TI region very similar to that of the previously reported FluA-20 (28) and S5V2-29 (29) mAbs (Figure 2, D-F). Antibodies H5.31 and H5.28 are clonally related siblings from 1 human subject with identical HCDR3 sequences and only several amino acid variations in their light and heavy chains. The overlay of the 2 crystal structures showed that H5.28 binds to the HA head domain in the same general manner as H5.31 (Figure 2F). The superposition of the variable domain and HA head domain of H5.31-HA onto those of $\mathrm{H} 5.28$-HA results in a $\mathrm{Ca}$ root-mean-square deviation (RMSD) of only $0.71 \AA$. The epitope recognized by $\mathrm{H} 5.31 / \mathrm{H} 5.28$ on VN/0 4 maps onto $1 \mathrm{HA} 1$ protomer of the H5N1 HA trimer (Protein Data Bank ID: 4BGW; Figure 2G). This epitope can be divided into 2 regions: the 220 loop of the receptor-binding domain (residues 217-224, and residue 229, H3 structure numbering) and a second region at the 90 loop (Figure $2 \mathrm{G})$. The sequences of the 220 loop of influenza A HA are relatively conserved, thus recognition of this region by H5.31/H5.28 partly explains the binding breadth of the 2 mAbs. In addition, the epitope recognized by $\mathrm{H} 5.31$ and $\mathrm{H} 5.28$ is inaccessible for mAbs to bind in the closed HA trimeric form (Figure $2 \mathrm{G}$ ). If we superimpose the head domain of H5.31/H5-HA complex onto H5N1 HA trimer (Protein Data Bank ID: 4BGW), the H5.31 heavy chain variable domain would occupy the space of the head domain of another adjacent HA protomer, e.g., in the closed trimer the head domain of an adjacent HA1 protomer clashes with the variable domain of $\mathrm{H} 5.31$ when bound.

In the H5.31/H5-HA structure, mAb H5.31 interacts with the HA 220 loop using HCDR3 and LCDR2 residues (Figure 2H). There are 6 hydrogen bonds (H-bond) or salt bridges between the 220 loop and the HCDRs. The highly conserved HA 220 loop residue R229 forms a salt bridge with the mAb H5.31 HCDR3 residue E98. Notably, LDCR2 residue Y49 forms an H-bond with the side chain of HCDR3 residue E98, assisting E98 to be well-positioned to interact with the 220 loop residue R229. All of those H-bonds are formed between the 220 loop main chain oxygen or nitrogen atoms and side chains of the mAb, and consequently this mode of $\mathrm{H}$-bond formation may contribute to the great breadth of the $\mathrm{mAb}$. A hydrophobic interaction between mAb H5.31 residue L100 and the 220 loop residue V223, and cation- $\pi$ interaction between 220 loop R220 and HCDR3 F96, also may contribute to the tight binding of H5.31. In addition, the tip of the elongated HCDR3 makes more contact with the 90 loop epitope, in which residue L100a (Kabat numbering) seems to play the major role (Figure 2I). The L100a side chain is surrounded by a hydrophobic pocket and its main chain nitrogen forms an H-bond with the HA G100 main chain oxygen. Peripheral to these L100a-Ag interactions, there are several polar interactions, such as a polar interaction between the HA D95 residue side chain and the HCDR3 G100c main chain nitrogen and salt bridge between D101 (HA) and R99 (HCDR3). In summary, H5.31 recognizes the HA head domain mainly by interacting with the HA 220 loop. Interestingly, the heavy chain DE loop in the framework region 3 (FR3) of H5.31 has a potential glycosylation site at residue N74 (Kabat numbering, sequence motif: $\mathrm{N}^{74} \mathrm{ASN}^{77}$ ), and 2 NAG residues can be fit into electron density around residue N74. Therefore, H5.31 is glycosylated in FR3, but without apparent functional alternation due to this modification.

Since the H5.28, H5.31, Flu-A20, and S5V2-29 mAbs are encoded by light chains with common features, we tested the hypothesis that a sequence signature associated with use of this gene could be used to identify new TI-specific antibodies. We studied the response of an otherwise healthy subject with exposure to diverse influenza vaccines who presented with acute laboratory-confirmed H3N2 virus respiratory infection in August 2017, and from whom we had previously isolated the TI mAb FluA-20 (28). For comparative purposes, we used deep sequencing to profile the $\mathrm{B}$ cell repertoire of this individual at various time points before or after natural infection. Sequencing time points included both healthy state baselines as well as responses to influenza vaccination (Figure $3 \mathrm{~A})$. At a time point approximately 1 week into the natural H3N2 infection, we obtained PBMCs, isolated plasmablasts (Supplemental Figure 4), and performed single-cell sequencing of expressed paired heavy and light chain mRNA $\left(\mathrm{sc}-\mathrm{V}_{\mathrm{H}} / \mathrm{V}_{\mathrm{L}} \mathrm{Seq}\right)$ on approximately 20,000 plasmablasts. We synthesized cDNA from a subset of recovered pairs of antibody genes and expressed the heavy-light chain pairs individually in small-scale $\mathrm{CHO}$ cell culture and then 

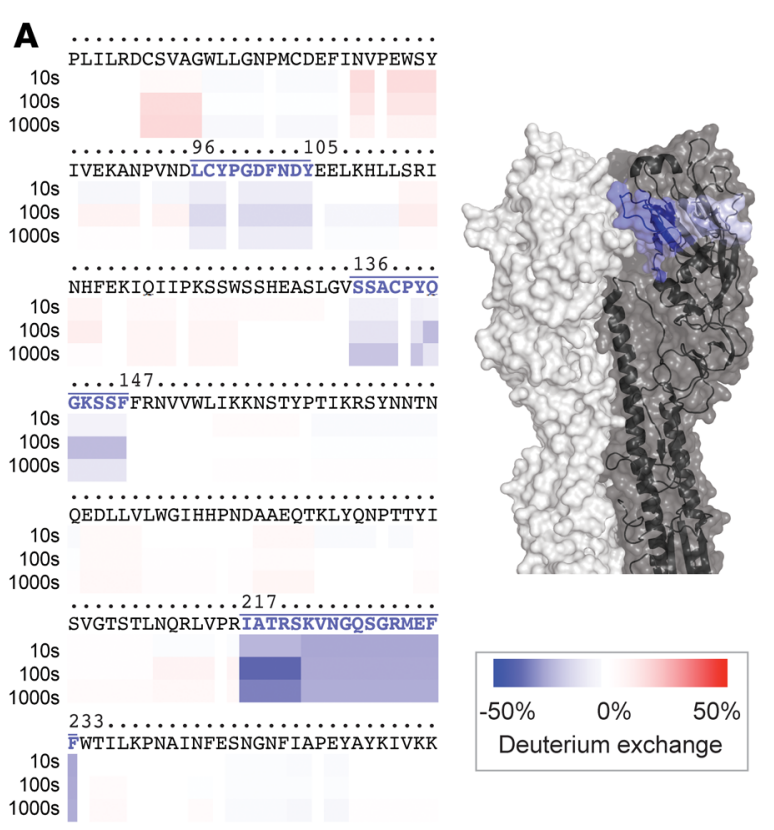

B
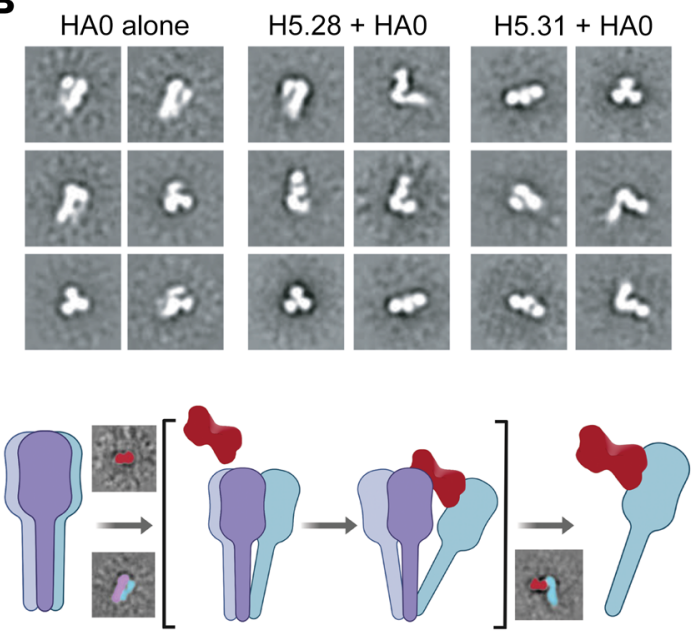

C

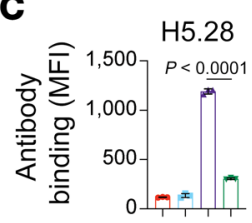

|

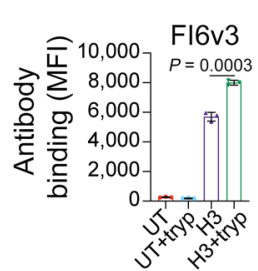

D $\mathrm{H} 5.31+\mathrm{H} 5 \mathrm{HA}$ complex

E

H5.28 + H5 HA complex

F Overlay of the two complexes
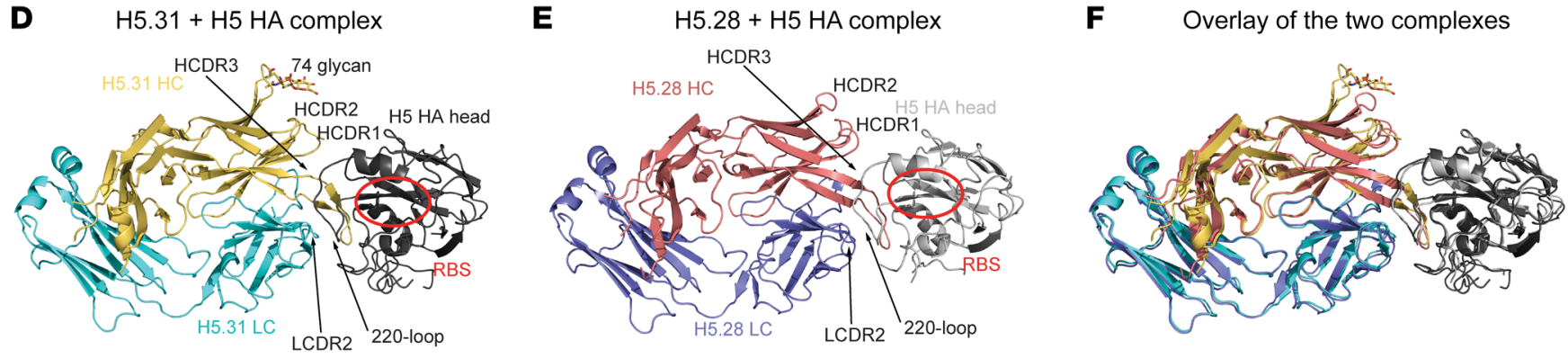

G

H

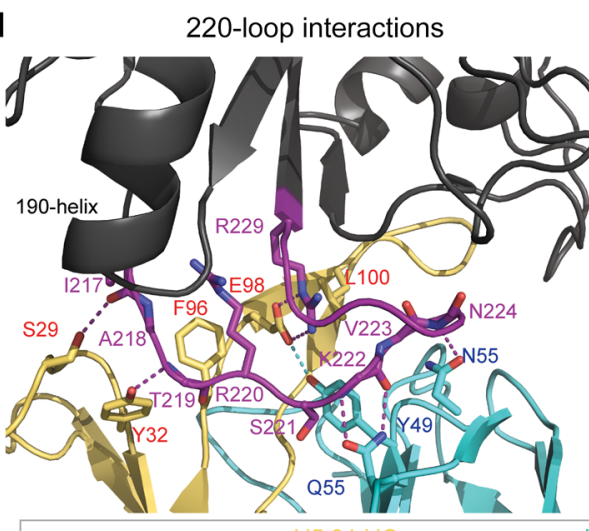

I 90-loop interactions
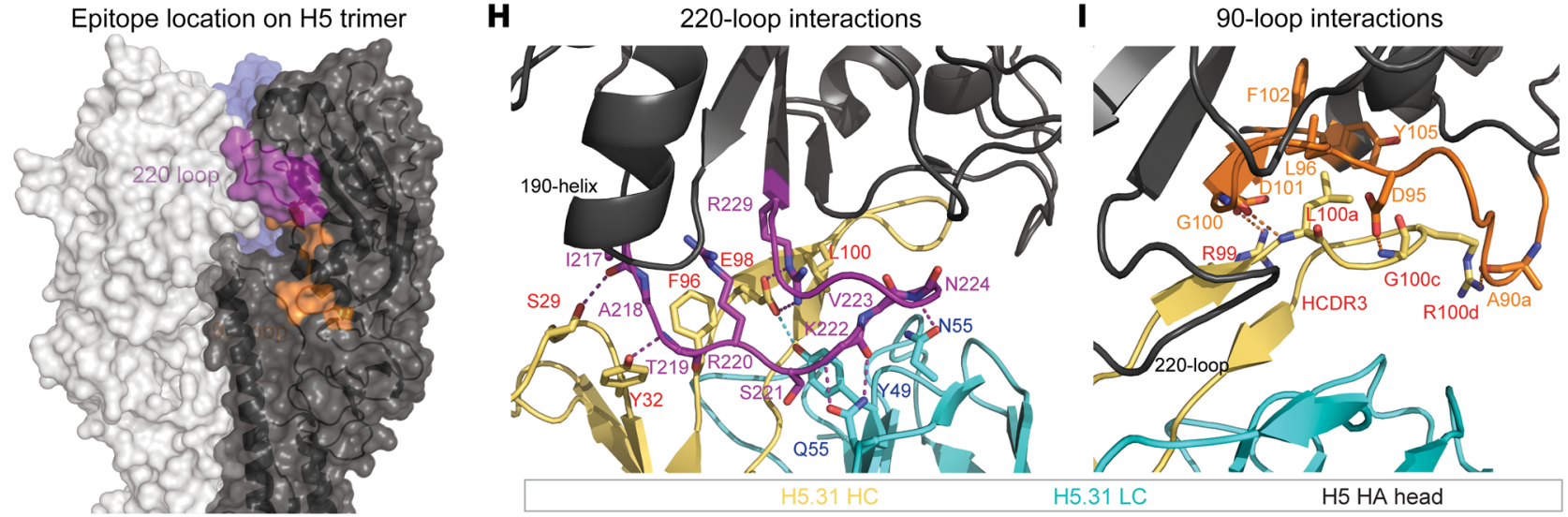
Figure 2. Structural and functional characterization of mAb H5.28 and H5.31 binding to HA. (A) HDX-MS with H5.28 Fab and H5 HA head domain identified a putative epitope for $\mathrm{H} 5.28$ at the TI. The amino acid sequence of the $\mathrm{H} 5$ head domain is shown with a ribbon diagram indicating differences in deuterium uptake. Blue colors indicate slower deuterium exchange in the presence of H5.28 Fab, while red colors indicate faster deuterium exchange in the presence of H5.28 Fab. Data are shown for 10 seconds, 100 seconds, and 1000 seconds of deuterium labeling. The 3 major peptides are colored blue on an $\mathrm{H} 5$ trimer. (B) Selected 2D class averages of H1 HA trimer (A/California/04/2009) alone or after a 20-second incubation with H5.28 (middle) or H5.31 Fab (right). All of the Fabs complexed with HA were in monomeric form, while a few apo HA trimers were observed. Below is a cartoon illustration showing that $\mathrm{H} 5.28$ or $\mathrm{H} 5.31 \mathrm{Fab}$ (magenta) results in dissociation of recombinantly expressed HA trimer (gray/blue/lavender), as visualized in the negative-stain EM data. (C) H5.28 and H5.31 preferentially bound to uncleaved HA expressed on the cell surface. In contrast, a recombinant form of the stem mAb FI6v3 preferentially bound to cleaved HA. $P$ values for the comparison between binding to cleaved and uncleaved HA were computed using an unpaired 2-way Student's $t$ test. (D) Crystal structure of $\mathrm{H} 5.31$ in complex with VN/04 HA. H5.31 heavy chain is colored in gold, the light chain in cyan, and H5 head domain in dark gray. A glycosylation site on H5.31, N74 on the H5.31 DE loop, is labeled and shown as sticks, and 2 fitted NAC residues of the glycan are shown also as sticks. The 220 loop of the VN/04 HA head domain is labeled, and the receptor binding site is highlighted with a red circle. (E) Crystal structure of H5.28 in complex with VN/04 HA. H5.28 heavy chain is colored in salmon, the light chain in lavender, and H5 HA head domain in light gray. (F) Superimposition of the 2 complex structures. Structural variations can be seen at HCDR1s, HCDR2s, and heavy chain DE loops. (C) The HA epitope recognized by H5.31 is mapped onto the surface of one protomer of the VN/04 HA trimer. The mapped protomer is colored dark gray, the 220 loop in purple, and the 90 loop in orange. The other 2 protomers are colored in light gray and blue, respectively. (H and I) Structural details of the H5.31/VN/O4-HA complex. (H) Interactions of H5.31 with 220 loop of the HA head domain, with the HCDR3 E98/LCDR2 Y49 interaction shown with blue dashes. Relevant residues of the HA head domain are labeled in purple, those of the heavy chain in red, and those of the light chain in blue. (I) Interactions of H5.31 HCDR3 with 90 loop and its C-terminal $\beta$ strand. Relevant residues of the HA head domain are labeled in orange, those of the heavy chain in red, and those of the light chain in blue.

purified IgG from cell supernatants with protein $G$ affinity resin. Purified recombinant antibodies were tested by ELISA for binding to diverse HAs (Group 1: H1, H5; Group 2: H3, H7), and by neutralization of a representative $\mathrm{H} 3 \mathrm{~N} 2 \mathrm{WT}$ virus corresponding to a recent H3N2 vaccine strain (A/Texas/50/2012). Sixteen of the antibodies exhibited heterosubtypic reactivity (binding to more than $1 \mathrm{HA}$ subtype) and HA protein specificity. These heterosubtypic antibodies included somatic variants of the FluA-20 lineage. However, we also identified other heterosubtypic mAbs that used the same light chain (IGKV1-39) as FluA-20, H5.28, and H5.31. Sequence alignment for one of these new mAbs, designated FluA-151, showed that 7 additional members of the clonal lineage for that antibody (which we designated Sibs 1-7) were also present in the collection of over 4000 plasmablast paired heavy-light chain sequences (indicated as Sibs in Figure 3B). We searched for FluA-151-like sequences in the collection of all antibody repertoire sequences for that donor obtained over the 4-year period 2014-2018 and found 178 additional somatic variants of the heavy chain and 99 additional variants of the light chain (Figure 3B). We constructed lineages of the clonotype, showing all corresponding heavy and light chain sequences, indicating the year and day after vaccination for the sample from which the variant was obtained (Figure 3B). The lineage included a diverse range of sequences, and members of this lineage appeared over multiple years of responses to vaccination. We expressed FluA-151, its inferred unmutated common ancestor (UCA) (FluA-151 UCA), and the Sib 1 variant (FluA-151_Sib1) and tested the heterosubtypic breadth of these related antibodies. The UCA had a relatively broad pattern of binding, recognizing HAs from Group 1 and Group 2 (Figure 3C). The intermediate FluA-151 Sib1 acquired recognition of a 2019 H3N2 strain and improved the $\mathrm{EC}_{50}$ value of binding for most Group 2 strains. The fully mature FluA-151 mAb was even broader, acquiring binding capacity for $\mathrm{H} 5$ and H6. These data show that the founder clone of the lineage was influenza HA-reactive and had substantial heterosubtypic breadth, and somatic mutations that occurred during elaboration of the lineage further enhanced heterosubtypic breadth. Notably, both FluA-151 and FluA-20 did not exhibit detectable binding to the HA from A/New York/107/2003, which has a deletion in the 220 loop, indicating that FluA-151 was likely a TI mAb.
We used our knowledge of the contact residues of other TI mAbs to define FluA-151 residues critical for epitope recognition using mutagenesis $(28,29)$. We previously demonstrated that alanine substitutions at D98 of the FluA-20 heavy chain or Y49 and Q55 of the FluA-20 light chain abrogated binding of FluA-20. Introducing these same mutations into FluA-151 greatly reduced binding against all HAs tested (Figure 3D). FluA-20, FluA-151, S5V2-29, H5.28, and H5.31 all share a S53N somatic mutation in the LCDR2, and introducing the S53N substitution into the FluA151 UCA improved binding 5- to 10-fold against most HAs tested (Figure 3D), providing evidence that this substitution improves binding and explaining the convergent evolution of the S53N substitution across multiple TI mAb lineages isolated from multiple donors. In the background of the mature FluA-151 mAb, the N53A substitution had a modest impact on binding, with the exception of binding to the VN/04 HA (Figure 3D). These data indicate that in the mature form of the $\mathrm{mAb}$ the importance of N53-mediated interactions ranged from nonessential to critical depending on variations at the TI epitope between different strains. In contrast to the dramatic effect of the Y49A substitution, the more conservative Y49F substitution did not affect the binding to some HAs but dramatically reduced binding to $\mathrm{H} 5$ and $\mathrm{H} 6$ HAs. Taken together, these results identify that FluA-151 shares many critical contact residues with FluA-20 and demonstrates that the S53N mutation is a common solution to enhance binding of multiple TI mAbs.

We next assessed the ability of FluA-151 to protect mice from weight loss following viral challenge in a sublethal model using an A/California/04/2009 H1N1 virus. One day prior to infection, we passively transferred mice with either FluA-151 WT or a FluA-151 LALA-PG Fc variant in which 3 mutations ablate Fc effector function (ref. 31 and Figure $3 \mathrm{E}$ ). Mice that received the negative control mAb 2D22 lost more than 20\% initial body weight but subsequently recovered. In contrast, animals treated with the positive control anti-stem mAb MEDI-8852 were completely protected from weight loss. While FluA-151 WT protected mice from major weight loss, mice who received the FluA-151 LALA-PG variant lost significantly more weight (Figure 3E), indicating that in the case of FluA-151, Fc-mediated effector function likely mediates protection from severe weight loss associated with influenza A virus infection. 
A Exposure Sequencing

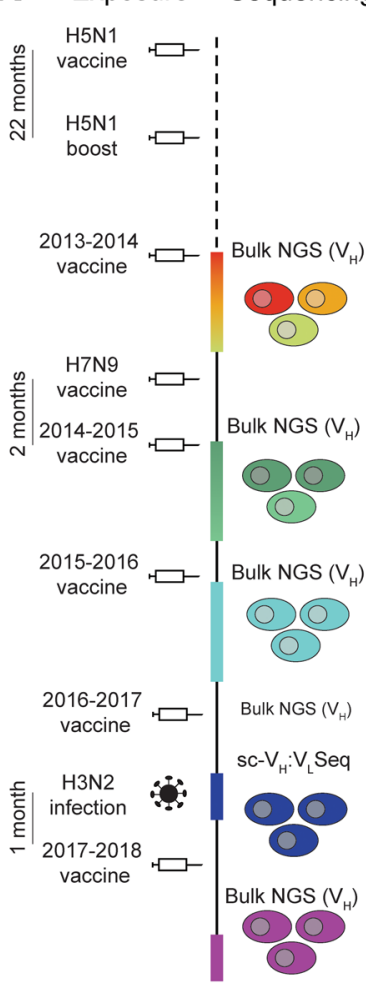

B

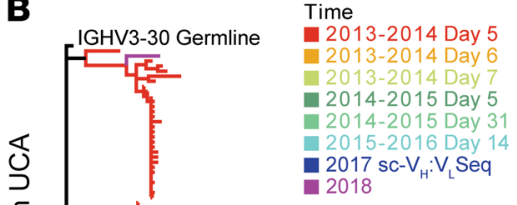

2018
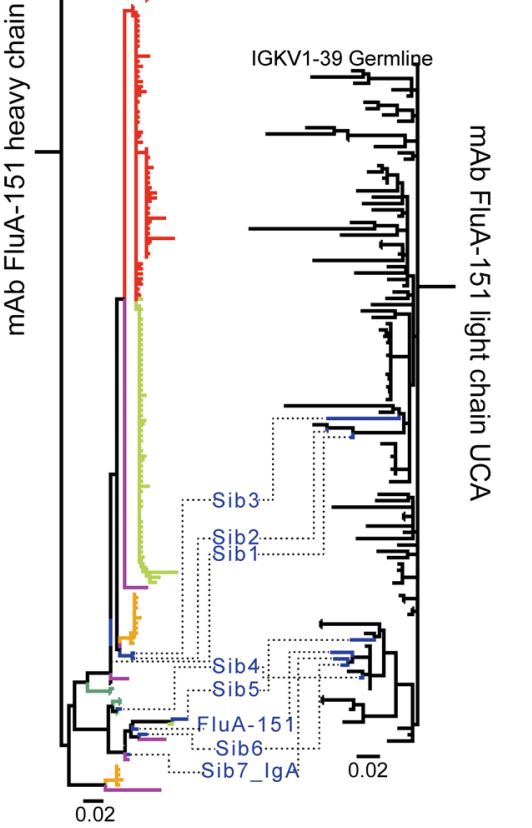

C Half-maximal effective concentration $\left(\mathrm{EC}_{50} ; \mathrm{ng} / \mathrm{mL}\right)$ New mAb lineage Controls

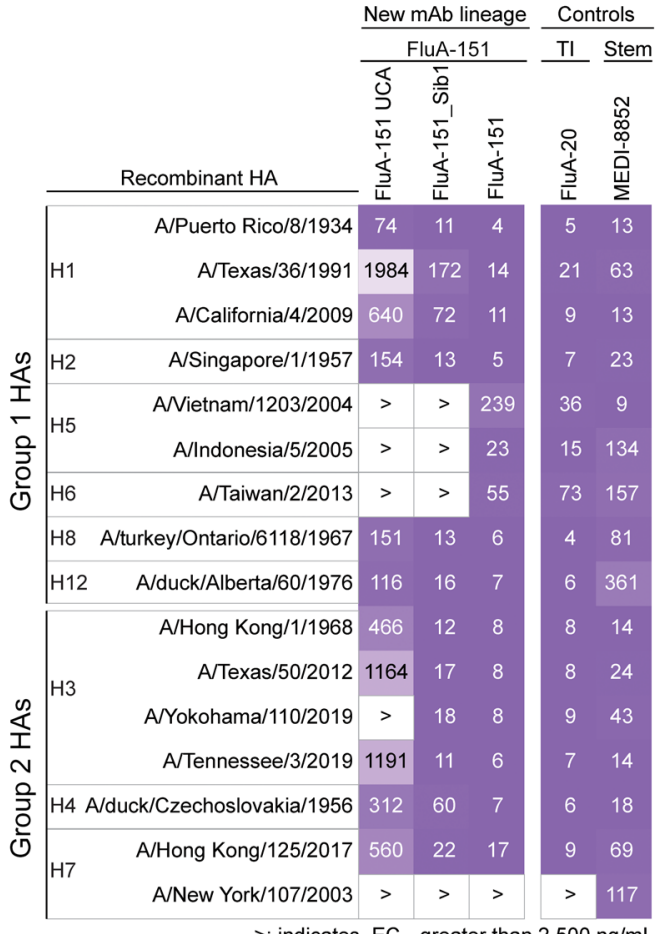

$>$; indicates $\mathrm{EC}_{50}$ greater than $2,500 \mathrm{ng} / \mathrm{mL}$
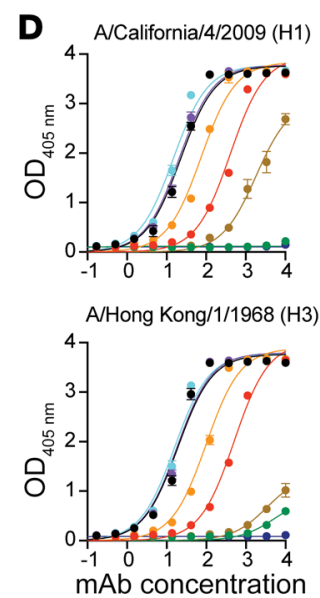

$\left(\log _{10} \mathrm{ng} / \mathrm{mL}\right)$
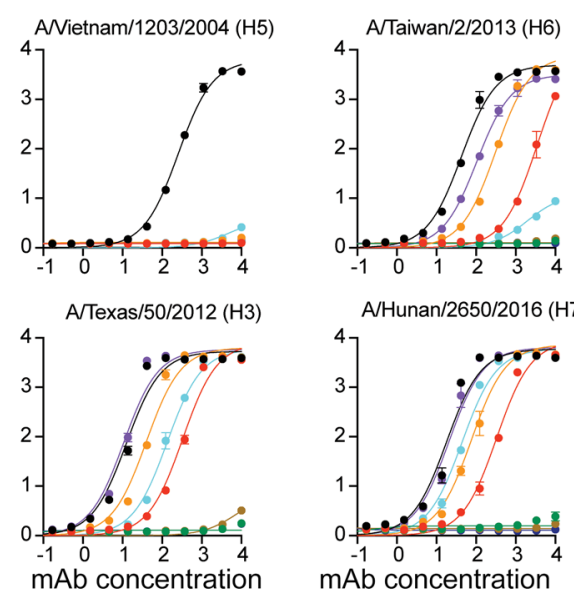

$\left(\log _{10} \mathrm{ng} / \mathrm{mL}\right)$

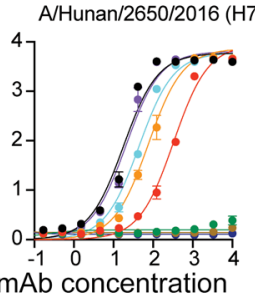

$\left(\log _{10} \mathrm{ng} / \mathrm{mL}\right)$
E

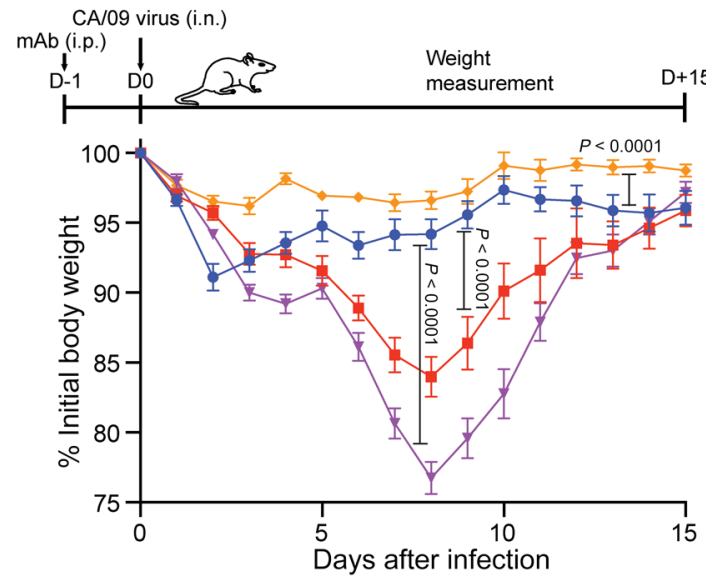

$\longrightarrow \quad$ FluA-151 WT $(10 \mathrm{mg} / \mathrm{kg})$
$\rightarrow-$ FluA-151 LALA-PG $(10 \mathrm{mg} / \mathrm{kg})$ $\rightarrow \quad 2 \mathrm{D} 22(10 \mathrm{mg} / \mathrm{kg})$

$\rightarrow-\operatorname{MEDI}-8852(10 \mathrm{mg} / \mathrm{kg})$

\begin{tabular}{|c|c|c|c|}
\hline & & & \\
\hline $\mathrm{HC}$ ) & A S53N (LC) & $\begin{array}{l}\rightarrow \text { Y49A (LC) } \\
\rightarrow \text { Y } 49 F(L C)\end{array}$ & $\rightarrow$ \\
\hline
\end{tabular}

Figure 3. Identification and functional characterization of a new TI mAb lineage recalled in the response to vaccination and natural infection. (A) Timeline showing the vaccination history of the research subject, with exposures and repertoire sequencing indicated. (B) Phylogenetic trees showing the FluA-151 lineage over 4 years of vaccination and infection, with branch colors corresponding to sequencing time point. At left, the heavy chain phylogeny for FluA-151 is color-coded based on year of vaccination and days postvaccination. At right, the light chain phylogeny for FluA-151 is shown. Paired heavy-light sequences identified by single-cell sequencing are shown with dashes connecting the heavy chain and light chain trees. (C) Binding of TI mAb FluA-151, a clonally related mAb (FluA-151_Sib1), and the inferred unmutated common ancestor of FluA-151 (FluA-151 UCA) to a panel of recombinant HAs. The previously described TI mAb FluA-20 and a recombinant version of the broadly reactive stem mAb MEDI-8852 are shown for comparison. FluA-20 and FluA-151 did not bind measurably to the A/New York/107/2003 HA, which has a 220 loop deletion. (D) ELISA binding of FluA-151 point mutants to HAs from different strains. Points and error bars represent the mean \pm SD of technical triplicates. Experiments were repeated twice, with data from one representative experiment shown. (E) FluA-151 protection from weight loss in a sublethal H1N1 challenge model. Mice were passively transferred with either FluA-151 WT (blue), FluA-151 LALA-PG (red), the positive control anti-stem mAb MEDI-8852 (orange), or the negative control anti-dengue mAb 2D22 (purple) 1 day prior to intranasal challenge with a sublethal dose of A/California/04/2009. For weight loss curves, error bars show the SEM. Statistical comparisons between treatment groups were performed using a repeated measurements 2-way ANOVA with Tukey's correction for multiple comparisons. 
Overlay of complexes for three mAbs

A
S

Figure 4. Structural and sequence alignments of TI mAbs reveal common features of epitope recognition. (A-C) Structural alignment of the Fab/HA head domain complexes of FluA-20, S5V2-29, and H5.31, with the HA head domains aligned to one another. (A) View of the structural alignment from the side (upper image) and top (lower image). (B) Despite differences in HCDR3 length, FluA-20, S5V2-29, and H5.31 all contact HA R229 using a D or E at heavy chain position 98. (C) FluA-20, S5V2-29, and H5.31 germline-encoded light chain residue $\mathrm{Y} 49$ makes hydrophobic contacts and a hydrogen bond with D98 or E98 of the heavy chain, while germline-encoded Q55 makes hydrogen bond contacts with the main chain amide and carbonyl groups of HA residue 222. The shared somatic mutation S53N introduces an additional hydrogen bond with the main chain amide of HA residue 224 . The interaction between HCDR3 D/E98 and LCDR2 Y49 is shown with blue dashes. (D) Sequence alignment of previously reported and newly reported TI mAbs identifies common recognition motifs, including a shared acidic residue at position 98 in the HCDR3, a common light chain (IGKV1-39), germline-encoded light chain contact residues shared by all mAbs, as well as a common light chain S53N somatic mutation. (E) Biolayer interferometry-based competition data demonstrate TI mAbs strongly compete with one another for binding to A/California/04/2009 HA trimer, but do not compete with the RBS mAb 5J8.

To further understand structural determinants of the molecular recognition of the TI site by the H5.31, FluA-20, and S5V2-29 antibodies, we overlaid the crystal structures of the 3 antigenantibody complexes. These complexes were remarkably similar on a global basis (Figure 4A). The critical contact residues for each of the 3 Fabs (each derived from independent subjects) were identical. All 3 Fabs shared a critical contact in the HCDR3 loop with an acidic ( $\mathrm{D}$ or $\mathrm{E}$ ) residue at Chothia position 98 contacting the R229 residue on HA (Figure 4B). The Fabs also shared 3 critical bonds on 2 residues made by the light chain, at HA residues 222 and 224 (Figure 4C). In addition, for all antibodies the light chain Y49 residue formed a hydrogen bond with the $\mathrm{D}$ or $\mathrm{E}$ residue at position 98 in the heavy chain (Figure $4 \mathrm{C}$ ). This interaction may be required for optimal alignment of residues for TI epitope recognition and might explain the effect of the Y49F substitution on the ability of FluA-151 to recognize some HAs (Figure 3D). Using this conserved interaction as a guide, we identified 2 additional putative TI antibodies, FluA-152 and FluA-153, from a paired sequencing data set of plasmablasts isolated approximately 1 week following PCR-confirmed H1N1 infection of an additional, independent donor. We aligned the antibody variable region sequences of FluA-20 (28), FluA-151, FluA-152, FluA-153, H5.28, H5.31, and the S5V2-29, S5V2-52, S1V2-37, and S1V2-58 antibodies (ref. 29 and Figure 4D) and observed a canonical sequence pattern, with a motif comprising (a) use of the IGKV1-39 light chain gene, (b) conservation of germline-encoded Y49 and Q55 residues in the light 
A

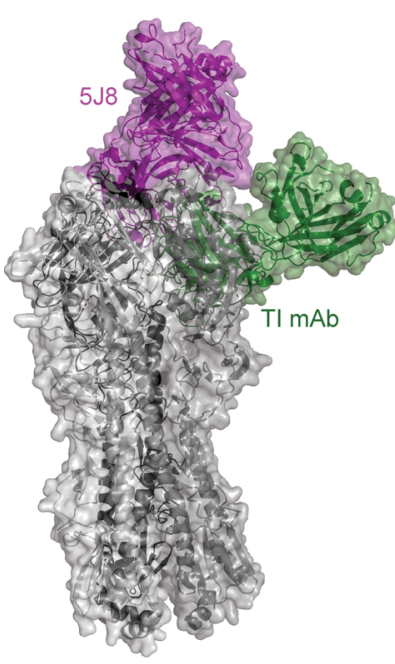

B
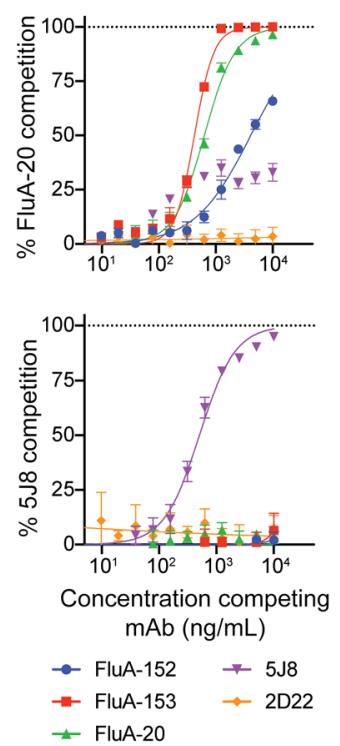
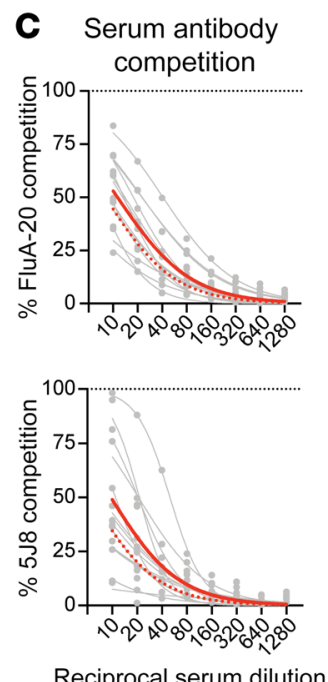

Reciprocal serum dilution

- Prevax average

- Postvax average

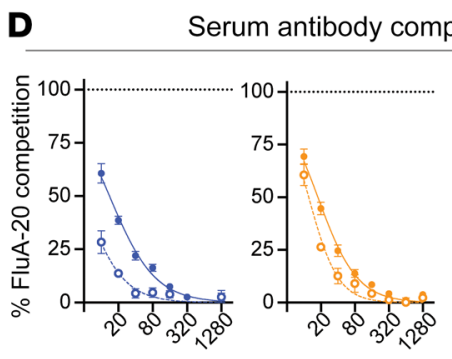

Serum antibody competition

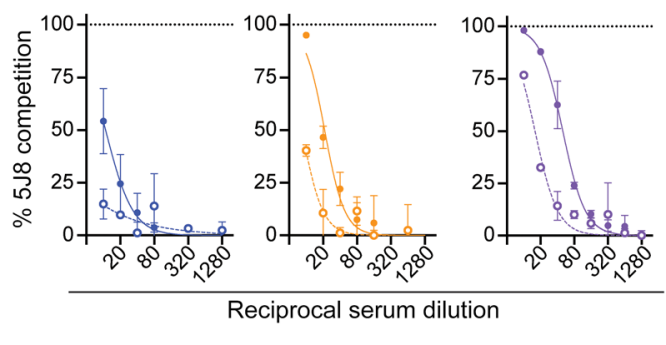

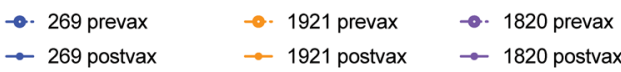

Figure 5. Measurement of serum antibody competition with HA head and HA TI mAbs in individuals before and after seasonal influenza vaccination. (A) Structure showing an H1N1 trimer with RBS-specific mAb 5/8 (purple) and TI mAb (green) binding sites indicated. (B) Competition ELISA binding curves with TI mAbs FluA-20, FluA-152, and FluA-153, the RBS mAb 5J8, and the negative control dengue virus mAb 2D22. After incubation with serial dilutions of the competing mAb or serum, biotinylated FluA-20 or 5J8 was added and competition was measured and normalized to a no-competition control. Competition with FluA-20 binding is shown in the top panel, and competition with 5J8 binding is shown in the bottom panel. (C) Competition ELISA binding curves for FluA-20 competition (top) and 5J8 competition (bottom) for human serum from 13 donors. Gray lines indicate competition levels in serum collected from individuals approximate a month after seasonal influenza vaccination. The dashed red line indicates the average of prevaccination competition with each reference $\mathrm{mAb}$, while the solid red line indicates the average of postvaccination competition. (D) Prevaccination and postvaccination serum competition with FluA-20 (top) and 5J8 (bottom) for 2 donors from whom TI mAbs were isolated (donors 269 and 1921) and 1 donor who had the highest level of competition with both 5J8 and FluA-20 (donor 1820). Competition binding experiments were performed for each sample in triplicate and repeated in at least 2 independent experiments. One representative experiment is shown. For all competition binding curves, data points indicate the mean and error bars indicate the SD.

chain, (c) introduction of an S53N somatic mutation in the light chain CDR2, and (d) inclusion of an acidic (D or E) residue in the HCDR3 at Chothia position 98 (which is encoded by nontemplated nucleotides in the $\mathrm{N} 1$ region of the $\mathrm{V}_{\mathrm{H}}-\mathrm{D}_{\mathrm{H}}$ gene junction added during recombination). Although FluA-20, S5V2-29, and H5.31 all shared these sequence features that mediate interactions with the HA 220 loop, S5V2-29 and H5.31 have longer HCDR3s and as a result make more contacts with the HA (Supplemental Figure 5). Clashes introduced by these additional contacts likely explain why these mAbs have more restricted reactivity. In contrast, mAbs FluA-20 and FluA-151 have shorter HCDR3s that likely avoid contacts with more variable $\mathrm{HA}$ residues.

We also considered that previous investigators had reported some human HA-specific antibodies identified by proteomic sequence analysis of serum antibodies that also displaced HA protomers during binding, indicating that these $\mathrm{mAbs}$ likely recognized an epitope at the interface between monomers (27). We aligned the amino acid sequences of those 3 antibodies (D1 H1-3/ H3-3, D1 H1-17/H3-14, and D2 H1-1/H3-1) and found that those sequences perfectly fulfilled the TI sequence motif described above (Figure 4D). These antibodies had been assigned putatively as binding to an occluded epitope near the TI region of the HA head domain based on a low-resolution electron microscopy (EM) structure and interference with binding of a trimer-specific antibody (ref. 27 and Supplemental Figure 6). However, recombinant forms of the D1 H1-3/H3-3, D1 H1-17/H3-14, and D2 H1-1/H3-1 antibodies competed for binding to trimeric HA with TI-specific antibodies but not with the receptor binding site-specific (RBS-specific) antibody 5J8 (Figure 4E). These mAbs also competed with TI mAbs for binding to a monomeric HA head domain (Supplemental Figure 6C). In addition, a separate group has recently described an antibody lineage with heterosubtypic breadth discovered using single B cell sequencing (32). This antibody, L3N6, also exhibits TI mAb sequence features (Figure $4 \mathrm{D}$ ) and competes for binding with known TI mAbs (Figure 4E). Together these data suggest these mAbs previously described by other groups also are in fact TI-specific antibodies.

Our recurrent identification of TI antibodies that share a common motif led us to hypothesize that antibodies targeting the TI antigenic site may be common in the serum antibody repertoire of many human donors. To test this hypothesis, we established a competition assay to measure serum antibody competition with either the TI mAb FluA-20 or the RBS-specific mAb 5J8, which target distinct antigenic sites (Figure 5A and Supplemental Table 2). We found that FluA-152 and FluA-153 competed with FluA-20 for binding, but did not prevent $5 \mathrm{~J} 8$ binding, confirming these mAbs also target the TI antigenic site (Figure $5 \mathrm{~B}$ ). We then tested the ability of serum collected from 13 individuals before and after seasonal influenza vaccination to compete with FluA-20 and 5J8. We observed competition with both FluA-20 and 5J8 in most 
donors tested, and for both reference antibodies we observed an increase in the level of competition following vaccination, indicating that vaccination boosted antibody levels in serum (Figure 5C). Importantly, we observed competition with FluA-20 in the serum of donor 269 (from whom FluA-20 and FluA-151 were isolated) as well as donor 1921 (from whom FluA-152 and FluA-153 were isolated; Figure 5D). The donor who had the highest level of FluA-20 competition following vaccination also had the highest level of 5J8 competition following vaccination (Figure 5D). Taken together, these results indicate that antibodies that compete with TI mAbs are common in serum of multiple individuals and can be present at levels comparable to that of antibodies that compete with RBS-directed antibodies targeting well-defined antigenic sites.

\section{Discussion}

In this work we use fine structural characterization of naturally occurring broad human mAbs that bind the HA TI to reveal canonical features of common human antibodies that bind a highly conserved site of vulnerability on influenza HA. These TI antibodies are common in human B cell repertoires, and they exhibit stereotypical features. Crystal structures of diverse TI mAbs with common light chain features reveal how the public clonotype we describe interacts with the highly conserved TI region, and we demonstrate that a shared somatic mutation improves binding, providing an explanation for the convergent evolution observed across $\mathrm{mAb}$ lineages isolated from multiple donors. We also demonstrate that previously described mAbs with broad reactivity recognize the same TI epitope using these shared sequence features. We note that previous investigators have determined the structure of H2214, a TI mAb that contacts many of the same HA residues but does not possess the canonical features we describe here (29), and that we and others have described antibodies that recognize the opposing face of the TI epitope and bind $\mathrm{H} 7$ and $\mathrm{H} 15$ HAs $(33,34)$. Thus, we conclude that there are additional modes of TI epitope recognition in addition to the common canonical motif we describe. When administered prophylactically or therapeutically, TI antibodies protect mice against challenge with diverse IAV strains (27-29), suggesting these mAbs may play a role in protecting individuals from severe disease during normal seasonal circulation of influenza A viruses. It is also likely that TI antibodies may form a considerable fraction of the cross-reactive response induced by some universal influenza vaccine approaches. Importantly, recent work analyzing the responses of individuals immunized with chimeric HA immunogens in a phase I clinical trial found that while immunization with chimeric HAs induced antibodies to HA stem epitopes, these vaccines also drove antibody responses to the TI epitope (35). It is possible that the accessibility of the canonical TI motif we describe here may explain why this response was observed across a number of individuals. While mass spectrometry approaches (27) and our serum antibody competition experiments suggest these TI mAbs are common in the serum of some individuals, quantifying the levels of these antibodies in humans and their contribution to protection is an important area of future work, particularly in the context of analyzing the human antibody response to candidate universal vaccines.

While these TI mAbs do not measurably neutralize virus in vitro, passive transfer of TI mAbs can prevent severe disease in ani- mal models, raising important questions about how this epitope is recognized in the context of an immune response. We showed that $\mathrm{HA}$ cleavage reduces $\mathrm{mAb}$ recognition of the TI epitope (Figure 2C; ref. 28), but other factors also can affect TI epitope accessibility. Previous work identified mouse mAbs that bound better to $\mathrm{HA}$ from acid-treated viruses, suggesting they recognized $\mathrm{pH}$-sensitive cryptic epitopes (36). One of these mAbs, Y8-10C2, binds an occluded epitope at the TI based on escape mutation epitope mapping (37). Importantly, Y8-10C2 also selected escape mutations in the HA stem region, demonstrating that mutations in this region likely affect trimer stability and epitope accessibility. We have previously described the structure of a mAb, H7.5, that partially displaces HA monomers to recognize a cryptic epitope near the TI, but binds in a different pose from the TI mAbs we describe here (38). Finally, others have demonstrated that HA hyperglycosylation focuses on mouse antibody responses to TI epitopes (39). Taken together, these findings indicate that (a) the HA is a less static structure than is often assumed, (b) multiple factors can influence the accessibility of TI epitopes, and (c) TI epitopes are commonly recognized during antibody responses induced by either natural infection or vaccination. Although TI mAbs lead to rapid dissociation of recombinant HA trimers in vitro, it is unclear how TI mAb binding alters the structure of HA trimers anchored in lipid bilayers, either on the surface of virions or on the cell membrane.

The work we present here also has implications for characterization of recurring structural motifs that facilitate antibody recognition of antigens. Historically, most B cell repertoire sequencing has focused on examination of the heavy chain, especially on the HCDR3 region, which dominates most antigen-antibody interactions. Instead of the typical heavy chain-driven interaction, the mode of molecular recognition for this class of antibodies may be determined instead mostly by canonical features of the light chain interaction, which is less commonly examined in immune repertoire sequencing efforts. Light chains do however modulate some antibody interactions with microbial pathogens. Importantly, recent work has identified a public clonotype targeting the Ebola virus glycoprotein (GP) that is elicited after natural infection or vaccination $(40,41)$. These GP-specific antibodies are encoded by IGHV3-15 and IGLV1-40, make conserved light chain and heavy chain contacts with GP, and have been isolated from multiple individuals. Remarkably, these Ebola-specific mAbs also exhibit convergent somatic mutations in the LCDR2 across multiple donors, suggesting that the TI epitope recognition motif we describe here may be one example of a broader class of public clonotypes in which both heavy chain and light chain motifs play important roles in epitope recognition. As paired sequencing of heavy and light chain variable genes in immune repertoires becomes increasing common, it is likely that similar public clonotypes that target epitopes from a variety of pathogens will be identified.

In summary, we identified the genetic and structural basis for recognition of the influenza virus HA head domain TI by human antibodies that are elicited in a diverse array of individuals. The sequence studies reveal a canonical motif comprising residues in the heavy and light chains from which we can infer TI-specificity. This TI class of antibodies exhibits broad heterosubtypic binding, and lineages of TI antibodies can acquire even broader or near universal recognition of influenza type A viruses. The common 
appearance of this class of antibody in diverse individuals suggests that the functional role of antibodies targeting the TI antigenic site in protection from seasonal influenza should be explored further.

Data availability. Atomic coordinates and structure factors for the crystal structures of H5.28 and H5.31 Fabs in their complexes with HA head domains have been deposited in the Protein Data Bank with the accession codes 6P3S (rH5.28 Fab in complex with H5 head domain), and 6P3R (rH5.31 Fab in complex with $\mathrm{H} 5$ head domain). The sequences of FluA-151, FluA-152, FluA-153, and FluA-151 sibling mAbs have been deposited to Genbank (accession numbers MZ328314-MZ328335).

\section{Methods}

Detailed experimental methods are available in the Supplemental Materials.

Statistics. The descriptive statistics mean \pm SEM or mean \pm SD were determined for continuous variables as noted. For the A/California/04/2009 lethal challenge model, survival curves were estimated using the Kaplan-Meier method and curves compared using the 2-sided log rank test (Mantel-Cox) with subjects right censored, if they survived until the end of the study. To correct for multiple comparisons, a Bonferroni-corrected threshold for statistical significance was determined $(P<0.006)$. For the A/California/04/2009 sublethal challenge model, statistical comparisons between the weight loss observed between treatment groups were performed using a repeated measurements 2-way analysis of variance (ANOVA) with Tukey's correction for multiple comparisons. Associated $P$ values are reported without defining a cutoff for statistical significance. For cell-surface display assays measuring binding of $\mathrm{mAbs}$ to cleaved and uncleaved HA, $P$ values for the comparison between binding to cleaved and uncleaved HA were computed using an unpaired 2-way Student's $t$ test and associated $P$ values are reported without defining a cutoff for statistical significance.

Study approval. All studies involving human subjects were approved by the Vanderbilt University Medical Center IRB, and peripheral blood was collected from donors following written informed consent. Demographic details of human donors used in this study are available in Supplemental Table 2. Animal studies were conducted in the AAALAC-accredited laboratories of Utah State University or Vanderbilt University Medical Center in accordance with the approval of the IACUCs of those institutions.

\section{Author contributions}

SJZ, JD, IMG, NJT, and JEC conceived and designed the research. SJZ, IMG, PG, NJT, NK, CS, ECC, and JEC isolated, sequenced, and analyzed antibodies. SJZ, IMG, PG, NJT, SB, and SL performed in vitro profiling of $\mathrm{mAb}$ activities. IMG, PG, and JEC designed and analyzed mouse studies on the in vivo activity of mAbs. PG, RPI, NS, and JBW performed mouse studies. JD determined the $\mathrm{X}$-ray structures of the Fabs and related complexes. SL performed the HDX-MS experiments. JAF, RB, CS, and ECC performed and interpreted sequencing experiments. RSN, RES, and RHC prepared recombinant antibodies and viral proteins. HLT and ABW performed EM studies. SJZ and JEC wrote the initial manuscript, and all authors edited and approved the final manuscript.

\section{Acknowledgments}

This work was supported by National Institutes of Health (NIH) grants U19 AI117905, U01 AI150739, and contract HHSN272201400024C. The project described was supported by a Clinical and Translational Science Award (no. UL1 TR002243) from the National Center for Advancing Translational Sciences (NCATS) (Vanderbilt Institute for Clinical and Translational Research, voucher no. VR53922). SJZ was supported by NIH training grant T32 AI095202. Vanderbilt University Medical Center has used the nonclinical and preclinical services program offered by the Division of Microbiology and Infectious Diseases (DMID) in the National Institute of Allergy and Infectious Diseases (NIAID). Funding for the H5.28 and H5.31 mouse studies research was provided by contract number HHSN272201700041I, task order A09, from the Respiratory Diseases Branch, DMID, NIAID, NIH. The contents of this publication are solely the responsibility of the authors and do not necessarily represent the official views of NCATS, NIAID, or NIH. X-ray diffraction data were collected at Beamline 21-ID-G at the Advanced Photon Source, a U.S. Department of Energy Office of Science User Facility operated for the Office of Science by Argonne National Laboratory under contract no. DE-AC02-06CH11357. Use of the LS-CAT Sector 21 was supported by the Michigan Economic Development Corporation and the Michigan Technology Tri-Corridor (grant 085P1000817). Support for crystallography was provided by the Vanderbilt Center for Structural Biology. We gratefully acknowledge the authors from the originating laboratories and submitting laboratories where genetic sequence data were generated and shared via the Global Initiative on Sharing All Influenza Data (42), with a list of authors available in Supplemental Table 3.

Address correspondence to: James E. Crowe Jr, Department of Pediatrics and Department of Pathology, Microbiology, and Immunology, Vanderbilt Vaccine Center, Nashville, Tennessee, USA. Phone 615.343.8064; Email: james.crowe@vumc.org.
1. Freidl GS, et al. Influenza at the animal-human interface: a review of the literature for virological evidence of human infection with swine or avian influenza viruses other than A(H5N1). Euro Surveill. 2014;19(18):20793.

2. Neumann G, Kawaoka Y. Transmission of influenza A viruses. Virology. 2015;479-480:234-246.

3. Nordin J, et al. Influenza vaccine effectiveness in preventing hospitalizations and deaths in persons 65 years or older in Minnesota, New York, and Oregon: data from 3 health plans. J Infect Dis. 2001;184(6):665-70.
4. Bridges CB, et al. Effectiveness and cost-benefit of influenza vaccination of healthy working adults: a randomized controlled trial. JAMA. 2000;284(13):1655-1663.

5. Carrat F, Flahault A. Influenza vaccine: the challenge of antigenic drift. Vaccine. 2007;25(39-40):6852-6862.

6. Erbelding EJ, et al. A universal influenza vaccine: the strategic plan for the National Institute of Allergy and Infectious Diseases. J Infect Dis. 2018;218(3):347-354.

7. Paules CI, et al. The pathway to a universal influ- enza vaccine. Immunity. 2017;47(4):599-603.

8. Ekiert DC, et al. Antibody recognition of a highly conserved influenza virus epitope. Science. 2009;324(5924):246-251.

9. Okuno Y, et al. A common neutralizing epitope conserved between the hemagglutinins of influenza A virus $\mathrm{H} 1$ and $\mathrm{H} 2$ strains. J Virol. 1993;67(5):2552-2558.

10. Corti D, et al. Heterosubtypic neutralizing antibodies are produced by individuals immunized with a seasonal influenza vaccine. J Clin Invest. 2010;120(5):1663-1673. 
11. Corti D, et al. A neutralizing antibody selected from plasma cells that binds to group 1 and group 2 influenza A hemagglutinins. Science. 2011;333(6044):850-856.

12. Dreyfus C, et al. Highly conserved protective epitopes on influenza B viruses. Science. 2012;337(6100):1343-1348.

13. Joyce MG, et al. Vaccine-induced antibodies that neutralize group 1 and group 2 influenza A viruses. Cell. 2016;166(3):609-623.

14. Kallewaard NL, et al. Structure and function analysis of an antibody recognizing all influenza A subtypes. Cell. 2016;166(3):596-608.

15. Smirnov YA, et al. An epitope shared by the hemagglutinins of $\mathrm{H1}, \mathrm{H} 2, \mathrm{H} 5$, and $\mathrm{H} 6$ subtypes of influenza A virus. Acta Virol. 1999;43(4):237-244.

16. Dreyfus C, et al. Structure of a classical broadly neutralizing stem antibody in complex with a pandemic $\mathrm{H} 2$ influenza virus hemagglutinin. JVirol. 2013;87(12):7149-7154.

17. Kashyap AK, et al. Combinatorial antibody libraries from survivors of the Turkish H5N1 avian influenza outbreak reveal virus neutralization strategies. Proc Natl Acad Sci U S A. 2008;105(16):5986-5991.

18. Kashyap AK, et al. Protection from the 2009 H1N1 pandemic influenza by an antibody from combinatorial survivor-based libraries. PLoS Pathog. 2010;6(7):e1000990.

19. Friesen RH, et al. A common solution to group 2 influenza virus neutralization. Proc Natl Acad Sci US A. 2014;111(1):445-450.

20. Ekiert DC, et al. A highly conserved neutralizing epitope on group 2 influenza A viruses. Science. 2011;333(6044):843-850.

21. Lang S, et al. Antibody $27 \mathrm{~F} 3$ broadly targets influenza A Group 1 and 2 hemagglutinins through a further variation in $\mathrm{V}_{\mathrm{H}} 1-69$ antibody orientation on the HA Stem. Cell Rep. 2017;20(12):2935-2943.

22. Caton AJ, et al. The antigenic structure of the influenza virus A/PR/8/34 hemagglutinin (H1 subtype). Cell.1982;31(2 pt 1):417-427.

23. Altman MO, et al. Lamprey VLRB response to influenza virus supports universal rules of immunogenicity and antigenicity. Elife. 2015;4:07467.

24. Gerhard W, et al. Antigenic structure of influenza virus haemagglutinin defined by hybridoma antibodies. Nature. 1981;290(5808):713-717.

25. Das SR, et al. Defining influenza A virus hemagglutinin antigenic drift by sequential monoclonal antibody selection. Cell Host Microbe. 2013;13(3):314-323.

26. Angeletti $D$, et al. Defining B cell immunodominance to viruses. Nat Immunol. 2017;18(4):456-463.

27. Lee J, et al. Molecular-level analysis of the serum antibody repertoire in young adults before and after seasonal influenza vaccination. Nat Med. 2016;22(12):1456-1464.

28. Bangaru S, et al. A site of vulnerability on the influenza virus hemagglutinin head domain trimer interface. Cell. 2019;177(5):1136-1152.

29. Watanabe A, et al. Antibodies to a conserved influenza head interface epitope protect by an IgG subtype-dependent mechanism. Cell. 2019;177(5):1124-1135.

30. Thornburg NJ, et al. Human antibodies that neutralize respiratory droplet transmissible $\mathrm{H} 5 \mathrm{~N} 1$ influenza viruses. JClin Invest. 2013;123(10):4405-4409.

31. Lo M, et al. Effector-attenuating substitutions that maintain antibody stability and reduce toxicity in mice. J Biol Chem. 2017;292(9):3900-3908.

32. Horns F, et al. Memory B cell activation, broad anti-influenza antibodies, and bystander activation revealed by single-cell transcriptomics. Cell Rep. 2020;30(3):905-913.

33. Yu F, et al. A potent germline-like human mono- clonal antibody targets a pH-sensitive epitope on H7N9 influenza hemagglutinin. Cell Host Microbe. 2017;22(4):471-483.

34. Dong J, et al. Anti-influenza H7 human antibody targets antigenic site in hemagglutinin head domain interface. J Clin Invest. 2020;130(9):4734-4739.

35. Nachbagauer R, et al. A chimeric hemagglutinin-based universal influenza virus vaccine approach induces broad and long-lasting immunity in a randomized, placebo-controlled phase I trial. Nat Med. 2021;27(1):106-114.

36. Yewdell JW, et al. Monoclonal anti-hemagglutinin antibodies detect irreversible antigenic alterations that coincide with the acid activation of influenza virus A/PR/834-mediated hemolysis. JVirol. 1983;48(1):239-248.

37. Yewdell JW, et al. Mutations in or near the fusion peptide of the influenza virus hemagglutinin affect an antigenic site in the globular region. JVirol. 1993;67(2):933-942.

38. Turner HL, et al. Potent anti-influenza H7 human monoclonal antibody induces separation of hemagglutinin receptor-binding head domains. PLoS Biol. 2019;17(2):e3000139.

39. Bajic G, et al. Influenza antigen engineering focuses immune responses to a subdominant but broadly protective viral epitope. Cell Host Microbe. 2019;25(6):827-835.

40. Ehrhardt SA, et al. Polyclonal and convergent antibody response to Ebola virus vaccine rVSV-ZEBOV. Nat Med. 2019;25(10):1589-1600.

41. Cohen-Dvashi H, et al. Structural basis for a convergent immune response against ebola virus. Cell Host Microbe. 2020;27(3):418-427.

42. Shu Y, McCauley J. GISAID: Global initiative on sharing all influenza data - from vision to reality. Euro Surveill. 2017;22(13):30494. 